\title{
EVOLVING FEATURES OF H $\beta$ DOPPLER VELOCITY FIELDS AT SITES OF FLARES
}

\author{
WEI LI, GUOXIANG AI AND HONGQI ZHANG \\ Beijing Astronomical Observatory, Chinese Academy of Sciences, \\ Beijing, 100080, China
}

\begin{abstract}
We analyzed eight active regions with more than 600 flare kernels and ribbons, and relevant time sequence $\mathrm{H} \beta$ chromospheric Dopplergrams. These data showed that during several hours prior to the flares, the velocity field evolves so that the sites of the flare kernels and ribbons become close to the inversion line of the velocity field. This result holds regardless of whether or not the flare sites are wholly located in blue-shifted areas, or are far from the the inversion line of the line-ofsight velocity field, or are partly within red-shifted areas.
\end{abstract}

\section{INTRODUCTION}

Many vector magnetograms and $\mathrm{H} \beta$ Dopplergrams have been obtained with the video vector magnetograph at the Huairou Solar Observing Station of the Beijing Astronomical Observatory. We have recently analyzed time sequences of $\mathrm{H} \beta$ Dopplergrams of 8 active regions containing more than 600 flare kernels and ribbons. The regions are AR5395 (1989 March), AR5629 (1989 August), AR5747 (1989 October), AR6001 (1990 April), AR6619 (1991 May), AR6818 (1991 September), AR6871 (1991 October), and AR6988 (1991 December). From analysis of these active regions, we confirmed the phenomenon that flares appear on the red-shifted side close to the inversion line of the $\mathrm{H} \beta$ Dopplergram (Ai et al. 1989). We also found some evolving features of $\mathrm{H} \beta$ Dopplergrams at flare sites several hours before the flares. In the following, we briefly describe these results using AR5629 as an example.

\section{Velocity Measurement Technique}

The method for measuring $\mathrm{H} \beta$ velocity fields has been described in detail in papers about the Solar Magnetic Field Telescope (Ai 1987). Briefly, the red and blue wings of the $\mathrm{H} \beta$ line ( $\pm 0.24 \AA$ from line center) are alternately selected by the filter pass-band by use of a KD*P electro-optical modulator at a frequency of $6.25 \mathrm{~Hz}$. The difference of the red and blue signals divided by their sum represents the Doppler shift. A CCD is the image detector and the calculation is done by an image processing computer. 


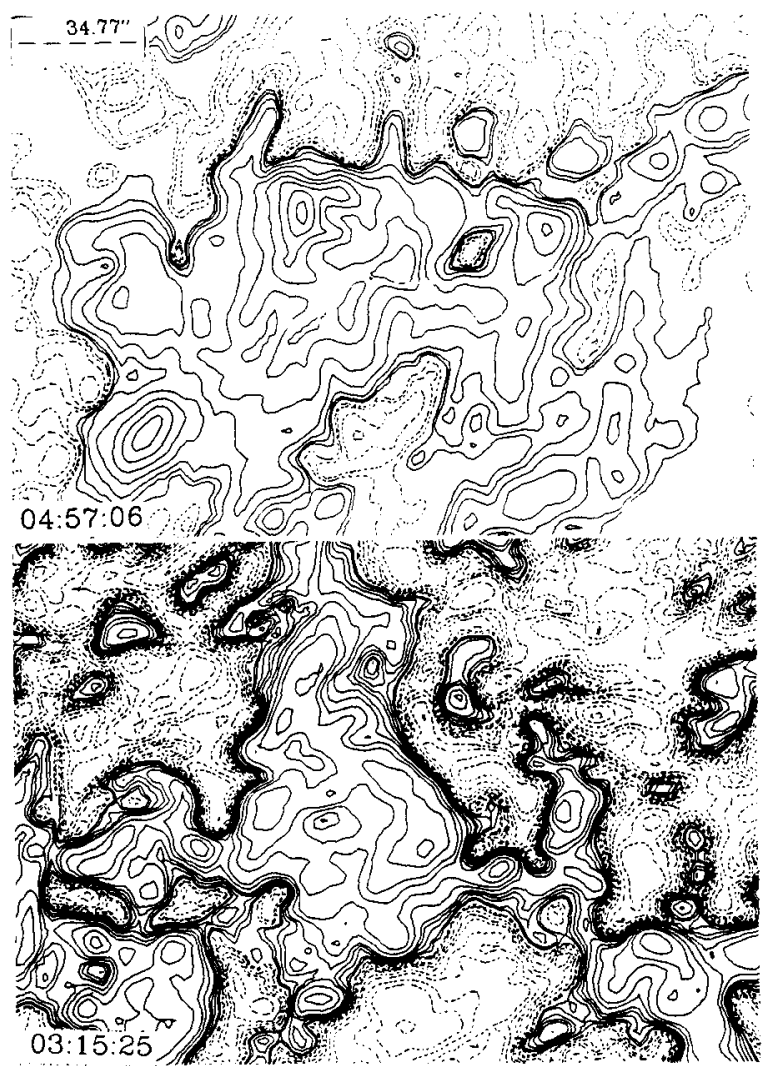

Fig. 1. Active region 5629 on 1989 August 12. North is up and west to the right. The top panel is a magnetogram at $0457 \mathrm{UT}$ with contours of $\pm 20,40,80,160,320,640,1280,1600,1920,2240,2560,2880 \mathrm{G}$. The lower panel is a Dopplergram at 0315 UT with solid (dashed) contours of downward (upward) flow at values of 50,100,200,300, 400,600, $800,1200,1600,2400,3200,4000,4800,5600,6400 \mathrm{~m} / \mathrm{s}$.

\section{OBSERVED PHENOMENA}

In general, locations of strong magnetic fields are associated with strong redshifted $\mathrm{H} \beta$ Doppler velocity fields (Figure 1). Red shifts are observed at the sites of flare kernels or ribbons for several hours before flares occur. Some of the flare kernels are located close to the inversion line of the line-of-sight $H \beta$ Doppler velocity field, while others are far from the inversion line - even at the centers of red-shifted regions. But, in both cases, the kernels move closer and closer to the inversion line of the velocity field as time goes on.

\section{Preflare blue shifts}

Blue-shifted areas near flare sites increase in number and strength prior to flares. In other words, newly formed blue-shifted areas appear in previously red-shifted 


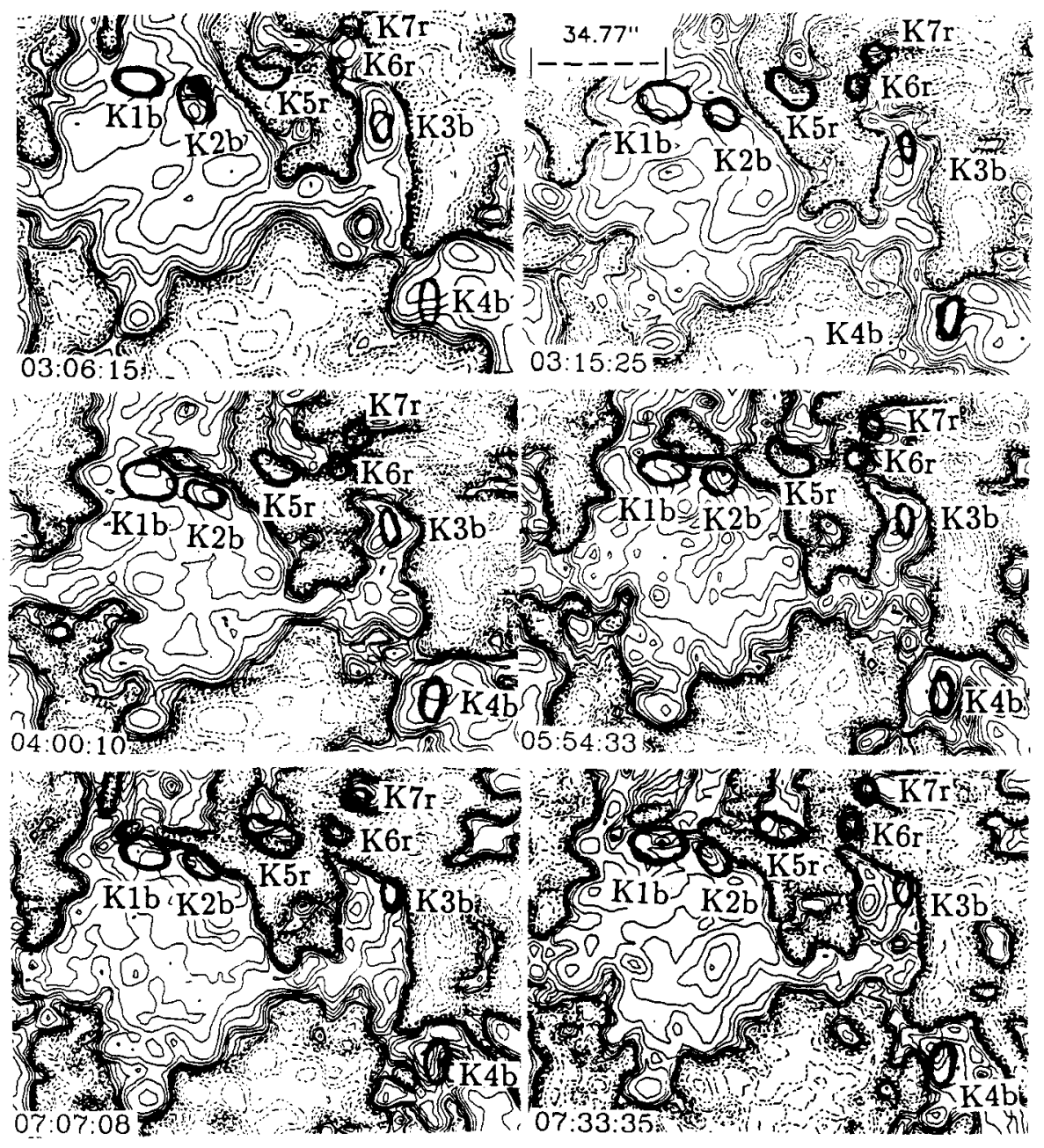

Fig. 2. A time sequence of $\mathrm{H} \beta$ Dopplergrams on 1989 August 12. The heavy solid lines indicate the positions of $\mathrm{H} \beta$ flare kernels of a flare that occurred at 0733 UT. Specifications are the same as for Figure 1. 
regions or existing blue-shifted regions become stronger near the sites of flares. For example, Figure 2 shows 1989 August 120733 UT H $\beta$ flare kernels K1b, $\mathrm{K} 2 \mathrm{~b}, \mathrm{~K} 3 \mathrm{~b}$, and $\mathrm{K} 4 \mathrm{~b}$ superimposed on a time series of Dopplergrams. These flare kernels were located on the centers of red-shifted areas of the Dopplergram at 0315 UT. At 0400 UT, a newly formed blue-shifted area appeared in the originally red-shifted region of the $\mathrm{H} \beta$ Dopplergram beside $\mathrm{K} 1 \mathrm{~b}$ and $\mathrm{K} 2 \mathrm{~b}$. At $0554 \mathrm{UT}$, the area of the new blue-shifted feature increased, but K3b and K4b were still located in the centers of red-shifted areas. At 0707 UT, another blueshifted area emerged beside $\mathrm{K} 3 \mathrm{~b}$. The kernel $\mathrm{K} 4 \mathrm{~b}$ had been located in the red-shifted area and near the inversion line of the $\mathrm{H} \beta$ velocity field.

\section{Preflare red shifts}

Red-shifted areas on the $\mathrm{H} \beta$ Dopplergrams, where flares will appear, steadily increase prior to the flares. The sites of some flare kernels and ribbons are locaied completely within blue-shifted features or are only partly located in redshifted features several hours before the flares. In the latter case, prior to flares, the size of the red-shifted area increases and in the former case a newly formed red-shifted area appears in the blue-shifted region. Thus, the flare sites become closer and closer to the inversion line of the $\mathrm{H} \beta$ velocity field. For examples, see kernels K5r, K6r, and K7r in Figure 2. At $0315 \mathrm{UT}$, K5 was wholly located in the blue-shifted area and $\mathrm{K} 6$, and $\mathrm{K} 7 \mathrm{r}$ were partly in the red-shifted area. At $0400 \mathrm{UT}$, the areas of red-shift in K6r and K7r increased. Kernel K5r was wholly in the blue-shifted area until $0554 \mathrm{UT}$. By $0707 \mathrm{UT}$, the K5r area was more than half filled with red-shifted flows. Kernels K5r, K6r, and K7r were located completely on the red-shifted side of the inversion line when the flares occurred at 0733 UT.

\section{CONCLUSION}

All the preflare red-shift values of the above flare kernels and ribbons are in the range of 0.1 to $6.6 \mathrm{~km} / \mathrm{s}$. The average value is about $1.9 \mathrm{~km} / \mathrm{s}$. Most values are concentrated in the range of 0.5 to $4 \mathrm{~km} / \mathrm{s}$. This is almost as the same as the solar rotational speed.

(1) As a result of evolving $\mathrm{H} \beta$ chromospheric Doppler velocity fields, the $\mathrm{H} \beta$ flares not only appear in red-shifted areas, but also are close to the inversion line of the $\mathrm{H} \beta$ line-of-sight velocity field. These observational phenomena indicate that the flare is produced in a place where the velocity field has a strong shear.

(2) The evolution of $\mathrm{H} \beta$ Doppler velocity fields before flares indicates that inflow, outflow, shearing and penetration of velocity fields contribute to the formation of flare, as do magnetic shear and magnetic squeeze.

\section{REFERENCES}

Ai, G. 1987, Publ. Beijing Astron. Obs., 9, 27

Ai, G., Zhang, H., Li, W., Li, J., \& Chen, J. 1989, Solar-Terrestrial Predictions: Proceedings of a Workshop at Leura, Australia October 16-20, 1989, 1, 264 